\begin{tabular}{|c|l|}
\hline Title & Remarks on analytic smoothing effect for the Schrödinger equation \\
\hline Author(s) & Ozawa, Tohru; Y amauchi, Kazuyuki \\
\hline Citation & Hokkaido University Preprint Series in Mathematics, 822, 1-16 \\
\hline Issue Date & 2006 \\
\hline DOI & 10.14943/83972 \\
\hline Doc URL & http://hdl.handle.net/2115/69630 \\
\hline Type & bulletin (article) \\
\hline File Information & pre822.pdf \\
\hline
\end{tabular}

Instructions for use 


\title{
Remarks on analytic smoothing effect for the Schrödinger equation
}

\author{
T. Ozawa ${ }^{1}$ and K. Yamauchi ${ }^{2}$ \\ Dedicated to Professor Masatake Miyake on the occation of his sixtieth birthday
}

\begin{abstract}
We study analytic smoothing effect of solutions to the Schrödinger equation with Cauchy data decaying exponentially at infinity. The domain of analyticity in the space variables of solutions is described under weight conditions on the data in the terms of the corresponding supporting functios. The domain of analyticity in the time variable is characterized by means of weight conditions of Gaussian type on the data. A generalization of various isometrical identities related to the analytic smoothing effect is introduced.
\end{abstract}

\section{Introduction}

We consider the regularity of wavefunctions given by solutions to the Cauchy problem for the free Schrödinger equation

$$
i \partial_{t} u+\frac{1}{2} \triangle u=0
$$

where $u: \mathbb{R} \times \mathbb{R}^{n} \ni(t, x) \mapsto u(t, x) \in \mathbb{C}, \partial_{t}=\partial / \partial t$, and $\triangle$ is the Laplacian in $\mathbb{R}^{n}$. For any Cauchy data $\phi \in L^{2}\left(\mathbb{R}^{n}\right)$ at $t=0$, the equation is solved as $u(t)=U(t) \phi$ by means of the free Schrödinger group $U(t)=\exp \left(i \frac{t}{2} \triangle\right)$ in $L^{2}\left(\mathbb{R}^{n}\right)$.

In this paper we study smoothing properties of the wavefunctions $u$ away from the initial time $t=0$. There is a large literature on this issue (see for instance [AHS], [HK1], [HK2], [HS1], [HS2], [MRZ], [Nak], [OYY], [RZ1], [RZ2], [T1], [T2] and references theirin). One of the basic tool is given by the generators of Galilei transformations $J=J(t)=x+i t \nabla=U(t) x U(-t)$. On the basis of the relation $J(t) U(t)=U(t) x$, we see that for any $\phi \in\langle x\rangle^{-1} L^{2}$ the corresponding solution $u(t)=U(t) \phi$ satisfies $\partial_{j} u(t) \in\langle x\rangle L^{2}$ for any $t \neq 0$, $j=1, \ldots, n$, where $\langle x\rangle=\left(1+x^{2}\right)^{1 / 2}, x^{2}=x_{1}^{2}+\cdots+x_{n}^{2}$ for $x=\left(x_{1}, \ldots, x_{n}\right)$.

\footnotetext{
${ }^{1}$ Department of Mathematics, Hokkaido University, Sapporo 060-0810, Japan

${ }^{2}$ Department of Business Administration, Faculty of Business Administration, Sapporo University, Sapporo 062-8520, Japan
} 
Similarly, for any $m \in \mathbb{N}$ we see that the condition $\phi \in\langle x\rangle^{-m} L^{2}$ implies that $\partial^{\alpha} u(t) \in\langle x\rangle^{|\alpha|} L^{2}$ for any $t \neq 0$ and any multi-index $\alpha$ with $|\alpha| \leq m$. This implies in particular that for any $\phi \in \bigcap_{m \geq 1}\langle x\rangle^{-m} L^{2}$ the corresponding solution $u(t)=U(t) \phi$ satisfies $u(t) \in \bigcap_{m \geq 1} H_{l o c}^{m} \subset C^{\infty}$ for any $t \neq 0$. This means that solutions become smooth instantly if the data decay faster than polynomials in the sense of $L^{2}$, even though there is no regularity in the data.

There arises a natural question what happens if the data decay exponentially in $L^{2}$. The first study in this direction was done by Hayashi and Saitoh [HS1] in one space dimension. Roughly speaking, they proved that for any data of exponential decay in $L^{2}$ the corresponding solution is analytic on a strip including the real line with width proportional to $|t|$ for $t \neq 0$ and that for any data of Gaussian decay the corresponding solution is entire for $t \neq 0$. The method of proof depends on a power series expansion.

The purpose of this paper is to generalize the theory in any space dimension by a different approach. Our method of proof depends on the Fourier-Laplace transform. As a result, a simple relation is given between domains of analyticity in space and exponential decay conditions on the Cauchy data in terms of corresponding supporting functions. Moreover, domains of analyticity in time are characterized on the basis of Gaussian decay conditions on the Cauchy data.

\section{Analyticity of solutions for Schrödinger equa- tions}

Our argument begins by describing analytic continuations of solutions for Schrödinger equations. For $t \in \mathbb{R}^{n}$ let $U(t)$ be the free propagator, i.e.

$$
U(t)=\exp \left(i \frac{t}{2} \triangle\right)=\mathfrak{F}^{-1} \exp \left(-i \frac{t}{2}|\xi|^{2}\right) \mathfrak{F},
$$

where $\mathfrak{F}$ denotes the Fourier transform. For $t \in \mathbb{R} \backslash\{0\}, U(t) \phi$ with $\phi \in L^{1}+L^{2}$ has the representation

$$
(U(t) \phi)(x)=(M(t) D(t) \mathfrak{F} M(t) \phi)(x),
$$

where $\mathfrak{F}$ is understood to be a bounded operator from $L^{1}+L^{2}$ to $L^{\infty}+L^{2}$,

$$
\begin{aligned}
& (M(t) \psi)(x)=\exp \left(\frac{i}{2 t}|x|^{2}\right) \psi(x), \\
& (D(t) \psi)(x)=(i t)^{-n / 2} \psi\left(t^{-1} x\right)
\end{aligned}
$$


with

$$
(i t)^{-n / 2}= \begin{cases}\left(\frac{1+i}{\sqrt{2}} \sqrt{t}\right)^{-n} & \text { if } t>0 \\ \left(\frac{1-i}{\sqrt{2}} \sqrt{-t}\right)^{-n} & \text { if } t<0 .\end{cases}
$$

(See $[\mathrm{C}]$, [SS], for example.)

It is therefore natural to define

$$
u(t, \zeta)=\exp \left(\frac{i}{2 t} \zeta^{2}\right) \cdot(i t)^{-n / 2}(\mathfrak{F} M(t) \phi)\left(\frac{\zeta}{t}\right)
$$

for $t \in \mathbb{R} \backslash\{0\}$ and $\zeta \in \mathbb{C}^{n}$ as an analytic continuation of $U(t) \phi$, where $\zeta^{2}=$ $\zeta \cdot \zeta=\sum_{j=1}^{n} \zeta_{j}^{2}$. To describe the analyticity of (2.1) in $\zeta$, we introduce

Definition 1 For any bounded convex set $\Omega \subset \mathbb{R}^{n}$ with $0 \in \operatorname{Int} \Omega$, its supporting function $\gamma_{\Omega}$ is defined by

$$
\gamma_{\Omega}(x)=\sup \{x \cdot p ; p \in \Omega\}
$$

for $x \in \mathbb{R}^{n}$.

Example $1 \gamma_{\Omega}(x)=a|x|$ for $\Omega=\left\{x \in \mathbb{R}^{n} ;|x| \leqq a,\right\}$.

$$
\gamma_{\Omega}(x)=a \sum_{j=1}^{n}\left|x_{j}\right| \text { for } \Omega=\left\{x \in \mathbb{R}^{n} ;-a \leqq x_{j} \leqq a, j=1, \ldots, n\right\} .
$$

Proposition 1 Let $\Omega \subset \mathbb{R}^{n}$ be a bounded convex set satisfying $0 \in \operatorname{Int} \Omega$. Let $\phi$ satisfy $e^{\gamma_{\Omega}} \phi \in L^{1}+L^{\infty}$. Then for any $t \in \mathbb{R} \backslash\{0\}$, the function $\zeta \mapsto u(t, \zeta)$ defined by (2.1) is an analytic continuation of $U(t) \phi$ on $\mathbb{R}^{n}+i t(\operatorname{Int} \Omega)$.

Remark 1 If $e^{\gamma_{\Omega}} \phi \in L^{1}+L^{\infty}$, then $\phi \in L^{1}$. See the proof of Lemma 1 below.

Proposition 1 follows from the following lemma.

Lemma 1 Let $\Omega \subset \mathbb{R}^{n}$ be a bounded convex set with $0 \in \operatorname{Int} \Omega$ and let $\gamma_{\Omega}$ be the supporting function of $\Omega$. Suppose that $e^{\gamma_{\Omega}} \phi \in L^{1}+L^{\infty}$ and let $\mathfrak{F} \phi$ be the Fourier transform given by

$$
(\mathfrak{F} \phi)(\zeta)=(2 \pi)^{-n / 2} \int \exp (-i \zeta \cdot x) \phi(x) \mathrm{d} x,
$$

where $\zeta=\xi+i \eta \in \mathbb{C}^{n}$ and $\zeta \cdot x=\xi \cdot x+i \eta \cdot x \in \mathbb{C}$. Then $\mathfrak{F} \phi$ is analytic on $\mathbb{R}^{n}+i(\operatorname{Int} \Omega)$. 
Proof. Assume that $\zeta_{0}=\xi_{0}+i \eta_{0} \in \mathbb{R}^{n}+i(\operatorname{Int} \Omega)$ and that $\delta>0$ satisfies $B\left(\eta_{0} ; \delta\right) \subset \operatorname{Int} \Omega$. Then, $\eta+\frac{\delta}{2|x|} x \in \Omega$ for each $x \in \mathbb{R}^{n} \backslash\{0\}$ and $\eta \in B\left(\eta_{0}, \delta / 2\right)$. As $(\eta+(\delta / 2|x|) x) \cdot x \leqq \gamma_{\Omega}(x)$ by definition, we see that

$$
\eta \cdot x \leqq \gamma_{\Omega}(x)-(\delta / 2)|x|
$$

for any $x \in \mathbb{R}^{n}$. Therefore

$$
\begin{aligned}
\left|x_{j} \exp (-i \zeta \cdot x) \phi\right| & \leqq|x| \exp (\eta \cdot x)|\phi| \\
& \leqq|x| \exp \left(\gamma_{\Omega}(x)-(\delta / 2)|x|\right)|\phi| \\
& \leqq|x| \exp (-(\delta / 2)|x|)\left|e^{\gamma_{\Omega}} \phi\right| \in L^{1}
\end{aligned}
$$

since $e^{\gamma_{\Omega}} \phi \in L^{1}+L^{\infty}$ and $|x| \exp (-(\delta / 2)|x|) \in L^{1} \cap L^{\infty}$. Similarly, $\exp (-i \zeta$. $x) \phi \in L^{1}$. By differentiating under the integral sign,

$$
\frac{\partial}{\partial \bar{\zeta}} \mathfrak{F} \phi=0
$$

around $\zeta_{0}$. By Looman-Menchoff's theorem (see [Nar] for example) $\mathfrak{F} \phi$ is analytic on $\mathbb{R}^{n}+i(\operatorname{Int} \Omega)$.

Remark 2 If $e^{a|x|^{2}} \phi \in L^{1}+L^{\infty}$ for some $a>0, e^{R|x|} \phi$ belongs to $L^{1}$ for any $R>0$. Lemma 1 ensures that $\mathfrak{F} \phi$ is an entire function provided $e^{a|x|^{2}} \phi \in$ $L^{1}+L^{\infty}$.

In the view of $(2.1)$, it is natural to define

$$
u(\tau, \zeta)=\exp \left(\frac{i}{2 \tau} \zeta^{2}\right) \cdot(i \tau)^{-n / 2}(\mathfrak{F} M(\tau) \phi)\left(\frac{\zeta}{\tau}\right)
$$

for $\tau \in \mathbb{C} \backslash\{0\}$ and $\zeta \in \mathbb{C}^{n}$ as an analytic continuation of $u(t, \zeta)$ defined by (2.1). In fact:

Proposition 2 Let $a>0$ and let $\phi$ satisfy $e^{a|x|^{2}} \phi \in L^{1}+L^{\infty}$. Then the function $(\tau, \zeta) \mapsto u(\tau, \zeta)$ is analytic on $\left(\mathbb{C} \backslash \overline{B\left(\frac{i}{4 a}, \frac{1}{4 a}\right)}\right) \times \mathbb{C}^{n}$, where

$$
\overline{B\left(\frac{i}{4 a}, \frac{1}{4 a}\right)}=\left\{\tau \in \mathbb{C} ;\left|\tau-\frac{i}{4 a}\right| \leqq \frac{1}{4 a}\right\} .
$$

Remark $3 u$ defined by (2.2) can be a double-valued function out of the factor $(i \tau)^{-n / 2}$ provided $n$ is odd. To be more specific, $U(t) \phi$ is connected with $-U(t) \phi$ through a mutual continuation on $\left(\mathbb{C} \backslash \overline{B\left(\frac{i}{4 a}, \frac{1}{4 a}\right)}\right) \times \mathbb{C}^{n}$ provided $n$ is odd.

For the proof of Proposition 2, we introduce 
Lemma 2 Let $\phi$ satisfy $e^{a|x|^{2}} \phi \in L^{1}+L^{\infty}$ for some $a>0$ and let

$$
(\Phi(\tau))(x)=\exp \left(i \tau|x|^{2}\right) \phi(x) .
$$

Then the function

$$
\{\tau \in \mathbb{C} ; \Im \tau>-a\} \times \mathbb{C}^{n} \ni(\tau, \zeta) \mapsto(\mathfrak{F} \Phi(\tau))(\zeta) \in \mathbb{C}
$$

is analytic.

Proof. As for the analyticity with respect to $\zeta$ we have only to check $e^{R|\cdot|} \Phi(\tau) \in$ $L_{x}^{1}$ for any $R>0$ because of Lemma 1 .

Let $\tau=t+i s$. Then,

$$
\begin{aligned}
e^{R|x|}(\Phi(\tau))(x) & =\exp \left(R|x|+i \tau|x|^{2}\right) \phi(x) \\
& =\exp \left(i t|x|^{2}\right) \cdot \exp \left(R|x|-(s+a)|x|^{2}\right) \cdot e^{a|x|^{2}} \phi(x) .
\end{aligned}
$$

Since $e^{a|x|^{2}} \phi \in L^{1}+L^{\infty}, e^{R|\cdot|} \Phi(\tau)$ belongs to $L_{x}^{1}$ provided $\Im \tau>-a$. Thus $\mathfrak{F}(\Phi(\tau))(\zeta)$ is analytic in $\zeta$.

The analyticity with respect to $\tau$ is given by the fact that $\frac{\partial}{\partial \bar{\tau}} \mathfrak{F}(\Phi(\tau))=0$, which is shown by differentiating under the integral sign. Here we use the estimate

$$
\begin{aligned}
& \left|\frac{\partial}{\partial t} \Phi(\tau)(x)\right|=\left|\frac{\partial}{\partial s} \Phi(\tau)(x)\right| \\
& \quad=\left.\left.|| x\right|^{2}(\Phi(\tau))(x)|\leqq| x\right|^{2} \exp \left((b-a)|x|^{2}\right) \cdot e^{a|x|^{2}} \phi(x)
\end{aligned}
$$

for $0<b<a$ and $\tau \in \mathbb{C}$ with $\Im \tau>-b$. Hence we have shown the analyticity of $\mathfrak{F}(\Phi(\tau))(\zeta)$.

Lemma 2 yields the following Lemma 3 since $\Im(1 / 2 \tau)>-a$ if and only if $|\tau-i /(4 a)|>1 /(4 a)$. Proposition 2 follows directly from Lemma 3.

Lemma 3 Let $e^{a|x|^{2}} \phi \in L^{1}+L^{\infty}$ for $a>0$. Then the function

$$
\left(\mathbb{C} \backslash \overline{B\left(\frac{i}{4 a}, \frac{1}{4 a}\right)}\right) \times \mathbb{C}^{n} \ni(\tau, \zeta) \mapsto(\mathfrak{F} M(\tau) \phi)\left(\frac{\zeta}{\tau}\right) \in \mathbb{C}
$$

is analytic.

Remark 4 It is possible to make $(\mathfrak{F} M(\tau) \phi)\left(\frac{\zeta}{\tau}\right)$ with $e^{a|x|^{2}} \phi \in L^{1}+L^{\infty}$ not be analytic on the outside of the domain mentioned in Lemma 3 . The integral

$$
\int_{0}^{\infty} \exp \left(-p y^{2}-q y\right) \mathrm{d} y
$$


converges if and only if $(p, q) \in\{(p, q) ; \Re p>0\} \cup\{(p, q) ; \Re p=0, \Im p \neq 0, \Re q \geq$ $0\} \cup\{(0, q) ; \Re q>0\}$. Hence, for $\phi_{a}(x)=e^{-a|x|^{2}}$,

$$
\left(\mathfrak{F} M(\tau) \phi_{a}\right)\left(\frac{\zeta}{\tau}\right)=\int_{\mathbb{R}^{n}} \exp \left(-i \frac{\zeta}{\tau} \cdot x+i \frac{|x|^{2}}{2 \tau}-\lambda|x|^{2}\right) \mathrm{d} x
$$

converges if and only if

$$
\begin{aligned}
(\tau, \zeta) & \in\left(\mathbb{C} \backslash \overline{B\left(\frac{i}{4 a}, \frac{1}{4 a}\right)}\right) \times \mathbb{C}^{n} \\
\cup & \left\{(t+i s, \xi+i \eta) ; \frac{s}{2\left(t^{2}+s^{2}\right)}=a, s \xi_{j}=t \eta_{j}, j=1, \ldots, n\right\} .
\end{aligned}
$$

Namely, $\left(\mathfrak{F} M(\tau) \phi_{0}\right)\left(\frac{\zeta}{\tau}\right)$ with $\tau \in \overline{B\left(\frac{i}{4 a}, \frac{1}{4 a}\right)}$ is not analytic even if it converges.

\section{An identity related to analytic solutions for Schrödinger equations}

We shall next consider identities by using the description of analytic continuations of $U(t) \phi$.

Theorem 1 Let $a>0$ and let $\phi$ satisfy $e^{a|x|^{2}} \phi \in L^{2}$. Then $U(t) \phi$ with $t \neq 0$ has an analytic continuation $u(t, \cdot)$ defined on $\mathbb{C}^{n}$, which satisfies that

$$
\begin{aligned}
& \left(2 \pi a t^{2}\right)^{-n / 2} \iint_{\mathbb{R}^{n} \times \mathbb{R}^{n}} \exp \left(-\frac{\eta^{2}}{2 a t^{2}}\right)\left|\exp \left(-i \frac{(\xi+i \eta)^{2}}{2 t}\right) u(t, \xi+i \eta)\right|^{2} \mathrm{~d} \xi \mathrm{d} \eta \\
= & \sum_{\alpha \geq 0} \frac{(2 a)^{|\alpha|}}{\alpha !}\left\|J^{\alpha} U(t) \phi\right\|_{L^{2}}^{2}=\left\|e^{a|x|^{2}} \phi\right\|_{L^{2}}^{2},
\end{aligned}
$$

where $J^{\alpha}=J^{\alpha}(t)=\prod_{k=1}^{n} J_{k}(t)^{\alpha_{k}}, J_{k}(t)=x_{k}+i t \partial_{k}$ and $\alpha=\left(\alpha_{1}, \ldots, \alpha_{n}\right)$ is a multi-index.

Proof. For the sake of Lemma 3 the function $U(t) \phi$ has an analytic continuation described by (2.2). Let $I$ be the LHS of (3.1), i.e.,

$$
I=\left(2 \pi a t^{2}\right)^{-n / 2} \iint_{\mathbb{R}^{n} \times \mathbb{R}^{n}} \exp \left(-\frac{\eta^{2}}{2 a t^{2}}\right)\left|\exp \left(-i \frac{(\xi+i \eta)^{2}}{2 t}\right) u(t, \xi+i \eta)\right|^{2} \mathrm{~d} \xi \mathrm{d} \eta .
$$

This equals

$$
\begin{aligned}
& (2 \pi a)^{-n / 2} t^{-2 n} \iint_{\mathbb{R}^{n} \times \mathbb{R}^{n}} \exp \left(-\frac{\eta^{2}}{2 a t^{2}}\right)\left|(\mathfrak{F} M(t) \phi)\left(\frac{\xi+i \eta}{t}\right)\right|^{2} \mathrm{~d} \xi \mathrm{d} \eta \\
& =(2 \pi a)^{-n / 2} \iint_{\mathbb{R}^{n} \times \mathbb{R}^{n}} \exp \left(-\frac{\eta^{2}}{2 a}\right)|(\mathfrak{F} M(t) \phi)(\xi+i \eta)|^{2} \mathrm{~d} \xi \mathrm{d} \eta .
\end{aligned}
$$


Let $\psi(t)=M(t) \phi$ and let $\Phi_{\varepsilon}\left(x, x^{\prime} ; \xi, \eta\right)$ be $(2 \pi)^{-3 n / 2} a^{-n / 2} \exp \left(-\varepsilon \xi^{2}-\frac{\eta^{2}}{2 a}-i \xi \cdot x+\eta \cdot x+i \xi \cdot x^{\prime}+\eta \cdot x^{\prime}\right) \psi(t)(x) \overline{\psi(t)\left(x^{\prime}\right)}$ where $\zeta \cdot \zeta^{\prime}=\sum_{k=1}^{n} \zeta_{k} \zeta_{k}^{\prime}$ for $\zeta=\left(\zeta_{1}, \ldots, \zeta_{n}\right), \zeta^{\prime}=\left(\zeta_{1}^{\prime}, \ldots, \zeta_{n}^{\prime}\right) \in \mathbb{C}^{n}$. These satisfy that

$$
\begin{aligned}
& \iint_{\mathbb{R}^{n} \times \mathbb{R}^{n}}\left(\iint_{\mathbb{R}^{n} \times \mathbb{R}^{n}} \Phi_{\varepsilon}\left(x, x^{\prime} ; \xi, \eta\right) \mathrm{d} x \mathrm{~d} x^{\prime}\right) \mathrm{d} \xi \mathrm{d} \eta \\
& =(2 \pi a)^{-n / 2} \iint_{\mathbb{R}^{n} \times \mathbb{R}^{n}} \exp \left(-\varepsilon \xi^{2}-\frac{\eta^{2}}{2 a}\right)|\mathfrak{F} \psi(\xi+i \eta)|^{2} \mathrm{~d} \xi \mathrm{d} \eta .
\end{aligned}
$$

This converges to $I$ as $\varepsilon \searrow 0$. Let the integral of (3.2) be denoted by $I_{\varepsilon}$.

As $\Phi_{\varepsilon}\left(x, x^{\prime} ; \xi, \eta\right)$ belongs to $L^{1}\left(\mathbb{R}^{4 n}\right), I_{\varepsilon}$ equals

$$
\int_{\mathbb{R}^{n}}\left(\int_{\mathbb{R}^{n}}\left(\int_{\mathbb{R}^{n}}\left(\int_{\mathbb{R}^{n}} \Phi_{\varepsilon}\left(x, x^{\prime} ; \xi, \eta\right) \mathrm{d} \eta\right) \mathrm{d} \xi\right) \mathrm{d} x^{\prime}\right) \mathrm{d} x
$$

by Fubini's theorem.

We remark that

$$
\begin{aligned}
\int_{\mathbb{R}^{n}} \exp \left(-\frac{\eta^{2}}{2 a}\right) e^{\eta \cdot\left(x+x^{\prime}\right)} \mathrm{d} \eta & =(2 a \pi)^{n / 2} \exp \left(\frac{a}{2}\left(x+x^{\prime}\right)^{2}\right) \\
& =(2 a \pi)^{n / 2} \exp \left(-\frac{a}{2}\left(x-x^{\prime}\right)^{2}\right) e^{a x^{2}} e^{a x^{\prime 2}}
\end{aligned}
$$

and that

$$
\int_{\mathbb{R}^{n}} \exp \left(-\varepsilon \xi^{2}\right) e^{-i \xi \cdot\left(x-x^{\prime}\right)} \mathrm{d} \xi=(\pi / \varepsilon)^{n / 2} \exp \left(-\frac{1}{4 \varepsilon}\left(x-x^{\prime}\right)^{2}\right) .
$$

These yield

$$
\begin{aligned}
& \int_{\mathbb{R}^{n}}\left(\int_{\mathbb{R}^{n}} \Phi\left(x, x^{\prime} ; \xi, \eta\right) \mathrm{d} \eta\right) \mathrm{d} \xi \\
& =(4 \pi \varepsilon)^{-n / 2} \exp \left(-\left(\frac{1}{4 \varepsilon}+\frac{a}{2}\right)\left(x-x^{\prime}\right)^{2}\right) e^{a x^{2}} \psi(t)(x) \overline{e^{a x^{\prime 2}} \psi(t)\left(x^{\prime}\right)} \\
& =\left(\frac{1}{4 \varepsilon}+\frac{a}{2}\right)^{-n / 2}(4 \varepsilon)^{-n / 2} \overline{W_{(1 / \varepsilon+2 a)^{-1}}\left(x-x^{\prime}\right) f(t)\left(x^{\prime}\right)} f(t)(x),
\end{aligned}
$$

where $f(t)(x)=e^{a x^{2}} \cdot \psi(t)(x)$ and $W_{\delta}$ denotes the Gauss-Weierstrass kernel, i.e.,

$$
W_{\delta}(x)=(4 \pi \delta)^{-n / 2} \exp \left(-\frac{x^{2}}{4 \delta}\right)
$$


(See $[\mathrm{M}]$ for example.) By setting $\delta(\varepsilon)=\left(\frac{1}{\varepsilon}+2 a\right)^{-1}$

$$
\begin{aligned}
I_{\varepsilon} & =(\delta(\varepsilon) / \varepsilon)^{n / 2} \int_{\mathbb{R}^{n}} \overline{W_{\delta(\varepsilon)} * f(t)(x)} \cdot f(t)(x) \mathrm{d} x \\
& =(\delta(\varepsilon) / \varepsilon)^{n / 2}\left\langle f(t), W_{\delta(\varepsilon)} * f(t)\right\rangle_{L^{2}} .
\end{aligned}
$$

Since $f(t) \in L^{2}$,

$$
\begin{aligned}
\lim _{\varepsilon \rightarrow 0} I_{\varepsilon} & =\lim _{\varepsilon \rightarrow 0}(\delta(\varepsilon) / \varepsilon)^{n / 2}\langle f(t), f(t)\rangle_{L^{2}} \\
& =\int_{\mathbb{R}^{n}}|f(t)(x)|^{2} \mathrm{~d} x \\
& =\int_{\mathbb{R}^{n}} e^{2 a x^{2}}|\phi(x)|^{2} \mathrm{~d} x .
\end{aligned}
$$

We thus have

$$
I=\int_{\mathbb{R}^{n}} e^{2 a x^{2}}|\phi(x)|^{2} \mathrm{~d} x,
$$

where the right hand side is rewritten as

$$
\begin{aligned}
\sum_{\alpha \geq 0} \frac{(2 a)^{|\alpha|}}{\alpha !} \int_{\mathbb{R}^{n}} x^{2 \alpha}|\phi(x)|^{2} \mathrm{~d} x & =\sum_{\alpha \geq 0} \frac{(2 a)^{|\alpha|}}{\alpha !}\left\|x^{\alpha} \phi\right\|_{L^{2}}^{2} \\
& =\sum_{\alpha \geq 0} \frac{(2 a)^{|\alpha|}}{\alpha !}\left\|U(t) x^{\alpha} U(-t) U(t) \phi\right\|_{L^{2}}^{2} \\
& =\sum_{\alpha \geq 0} \frac{(2 a)^{|\alpha|}}{\alpha !}\left\|J^{\alpha}(t) U(t) \phi\right\|_{L^{2}}^{2}
\end{aligned}
$$

This completes the proof.

\section{Generalization}

We shall generalize Theorem 1 by the following statement. It is convenient to use the notation

$$
\left(\mathfrak{L}_{\Omega}\right) p(x)=\int_{\Omega} p(y) e^{2 x \cdot y} \mathrm{~d} y .
$$

Theorem 2 Let $\Omega \subset \mathbb{R}^{n}$ be a convex open set with $0 \in \operatorname{Int} \Omega$. Suppose that $p \in L^{1}(\Omega)$ is non-negative and that

$$
\operatorname{supp} p \cap \Omega=\Omega .
$$


Suppose that $\mathfrak{L}_{\Omega} p \in L_{\text {loc }}^{1}$ and assume that $\left(\mathfrak{L}_{\Omega} p\right)|\phi|^{2} \in L^{1}$. Then, $U(t) \phi$ with $t \neq 0$ has an analytic continuation $u(t, \cdot)$ defined on $\mathbb{R}^{n}+i t \Omega$, which satisfies that

$$
\begin{aligned}
& |t|^{-n} \int_{t \Omega} q(\eta / t)\left(\int_{\mathbb{R}^{n}}\left|\exp \left(-i \frac{(\xi+i \eta)^{2}}{2 t}\right) u(t, \xi+i \eta)\right|^{2} \mathrm{~d} \xi\right) \mathrm{d} \eta \\
& \quad=\int_{\mathbb{R}^{n}}\left(\mathfrak{L}_{\Omega} q\right)(x)|\phi(x)|^{2} \mathrm{~d} x
\end{aligned}
$$

for any $q \in L^{1}(\Omega)$ with $|q| \leqq p$.

For the proof of this theorem we prepare two properties. In the following lemma we assume that $\Omega$ is a bounded convex open set with $0 \in \Omega$ and that $p$ is the same as above.

Lemma 4 (1) For $\rho>0$ let $\Omega_{\rho}$ be defined by the set

$$
\left\{y ; \frac{1}{\rho} y \in \Omega\right\} \text {. }
$$

Then, $\gamma_{\Omega_{\rho}}=\rho \gamma_{\Omega}$.

(2) Let $\Omega_{\rho}$ be the same as above. Then, for each $0<\delta<1$ there exists a positive constant $C=C(\delta)$ such that

$$
\mathfrak{L}_{\Omega} p \geqq C \exp \left(2 \gamma_{\Omega_{1-\delta}}\right) .
$$

Proof. The first part follows by the inequalities

$$
y \cdot x=\rho\left(\frac{1}{\rho} y \cdot x\right) \leqq \rho \gamma_{\Omega}(x) \quad \text { for } y \in \Omega_{\rho}
$$

and

$$
y \cdot x=\frac{1}{\rho}(\rho y \cdot x) \leqq \frac{1}{\rho} \gamma_{\Omega_{\rho}}(x) \quad \text { for } y \in \Omega
$$

(See [DS] for example.)

The second part is shown by the convexity of $\Omega$. In fact, by definition for any $x \in \mathbb{R}^{n}$ there is $y_{x} \in \Omega_{1-\delta / 2}$ such that

$$
(1-2 \delta / 3) \gamma_{\Omega}(x) \leqq y_{x} \cdot x \leqq(1-\delta / 2) \gamma_{\Omega}(x)
$$

for each $x \in \mathbb{R}^{n}$. Let $C_{1}=\sup \left\{\gamma_{\Omega}(-x) / \gamma_{\Omega}(x) ; x \neq 0\right\}$. Since

$$
\left\{\gamma_{\Omega}(-x) / \gamma_{\Omega}(x) ; x \neq 0\right\}=\left\{\gamma_{\Omega}(x) / \gamma_{\Omega}(-x) ; x \neq 0\right\},
$$


$C_{1}$ is greater than or equal to 1 . Let $\delta_{1}=\delta /\left(3 C_{1}\right)$ and assume that $z \in \Omega_{\delta_{1}}$. Then

$$
\begin{aligned}
\left(y_{x}+z\right) \cdot x & =y_{x} \cdot x-z \cdot(-x) \\
& \geqq(1-2 \delta / 3) \gamma_{\Omega}(x)-\delta_{1} \gamma_{\Omega}(-x) \\
& \geqq\left(1-2 \delta / 3-C_{1} \delta_{1}\right) \gamma_{\Omega}(x) \\
& =(1-\delta) \gamma_{\Omega}(x)=\gamma_{\Omega_{1-\delta}}(x) .
\end{aligned}
$$

The convexity of $\Omega$ yields

$$
y_{x}+\Omega_{\delta_{1}} \subset \Omega_{1-\delta / 2+\delta_{1}} \Subset \Omega .
$$

Therefore

$$
\begin{aligned}
\int_{\Omega} p(y) e^{2 x \cdot y} \mathrm{~d} y & \geqq \int_{\Omega_{\delta_{1}}} p\left(y_{x}+z\right) \exp \left(2\left(y_{x}+z\right) \cdot x\right) \mathrm{d} z \\
& \geqq \int_{\Omega_{\delta_{1}}} p\left(y_{x}+z\right) \exp \left(2 \gamma_{\Omega_{1-\delta}}(x)\right) \mathrm{d} z \\
& \geqq \exp \left(2 \gamma_{\Omega_{1-\delta}}(x)\right) \int_{\Omega_{\delta_{1}}} p\left(y_{x}+z\right) \mathrm{d} z
\end{aligned}
$$

A lower bound for the integral in the rightest hand side of (4.2) is essentially given by Fatou's lemma. Since the closure of $\Omega_{1-\delta / 2}$ is compactly included by $\Omega$, there are a sequence $\left\{y_{x_{j}}\right\} \subset \Omega_{1-\delta / 2}$ and $y_{0} \in \Omega$ such that

$$
\begin{aligned}
y_{x_{j}} & \rightarrow y_{0} \\
\int_{\Omega_{\delta_{1}}} p\left(y_{x_{j}}+z\right) \mathrm{d} z & \longrightarrow \inf \left\{\int_{\Omega_{\delta_{1}}} p\left(y_{x}+z\right) \mathrm{d} z ; x \in \mathbb{R}^{n}\right\} .
\end{aligned}
$$

Therefore for $0<\delta^{\prime}<\delta_{1}$ sufficiently large $j$ satisfies that $y_{x_{j}}-y_{0} \in \Omega_{\delta_{1}-\delta^{\prime}}$. By using the convexity of $\Omega$ this yields $y_{0}+\Omega_{\delta^{\prime}} \subset y_{x_{j}}+\Omega_{\delta_{1}}$ for sufficiently large $j$. Hence

$$
\int_{\Omega_{\delta_{1}}} p\left(y_{x_{j}}+z\right) \mathrm{d} z \geqq \int_{\Omega_{\delta^{\prime}}} p\left(y_{0}+z\right) \mathrm{d} z
$$

for sufficiently large $j$. Namely,

$$
\inf \left\{\int_{\Omega_{\delta_{1}}} p\left(y_{x}+z\right) \mathrm{d} z ; x \in \mathbb{R}^{n}\right\} \geqq \int_{\Omega_{\delta^{\prime}}} p\left(y_{0}+z\right) \mathrm{d} z .
$$

This with (4.2) yields

$$
\int_{\Omega} p(y) e^{2 x \cdot y} \mathrm{~d} y \geqq \int_{\Omega_{\delta^{\prime}}} p\left(y_{0}+z\right) \mathrm{d} z \cdot \exp \left(2 \gamma_{\Omega_{1-\delta}}(x)\right) .
$$


We have thus shown the second part of Lemma 4.

Proof of Theorem 2.

In the case that $\Omega$ is bounded, by the virtue of Lemma 4

$$
\exp \left(\gamma_{\Omega_{1-\delta}}\right) \phi \in L^{2}
$$

for $0<\delta<1$. Hence $U(t) \phi$ has an analytic continuation defined on $\mathbb{R}^{n}+i t \Omega$.

In the case that $\Omega$ is unbounded, Lemma 4 replaced $\Omega$ by $\Omega \cap B_{R}$ yields the analyticity of $U(t) \phi$ on $\mathbb{R}^{n}+i t \Omega \cap B_{R}$, where $B_{R}$ is the open ball with radius $R$ centered at the origin. Here we use

$$
\int_{\Omega} p(y) e^{2 x \cdot y} \mathrm{~d} y \geqq \int_{\Omega \cap B_{R}} p(y) e^{2 x \cdot y} \mathrm{~d} y .
$$

Namely,

$$
\left(\mathfrak{L}_{\Omega \cap B_{R}} p\right)|\phi|^{2} \in L^{1} .
$$

It is thus shown by the arbitrariness of $R$ that $U(t) \phi$ has an analytic continuation $u(t, \cdot)$ defined on $\mathbb{R}^{n}+i t \Omega$ if $\Omega$ is unbounded. We remark that $u(t, \zeta)$ can be described by (2.1) for $\zeta \in \mathbb{R}^{n}+i t \Omega$ even if $\Omega$ is unbounded.

We next show the identity in question. Let $I$ be the LHS of (4.1). By the representation of $u$

$$
\begin{aligned}
I & =|t|^{-n} \int_{t \Omega} q(\eta / t)\left(\int_{\mathbb{R}^{n}}\left|(i t)^{-n / 2}(\mathfrak{F} \psi(t))\left(\frac{\xi+i \eta}{t}\right)\right|^{2} \mathrm{~d} \xi\right) \mathrm{d} \eta \\
& =\int_{\Omega} q(\eta)\left(\int_{\mathbb{R}^{n}}|(\mathfrak{F} \psi(t))(\xi+i \eta)|^{2} \mathrm{~d} \xi\right) \mathrm{d} \eta,
\end{aligned}
$$

where $\psi(t)=M(t) \phi$. Let $\Psi_{\varepsilon}\left(x, x^{\prime} ; \xi, \eta\right)$ be

$$
(2 \pi)^{-n} q(\eta) \exp \left(-\varepsilon \xi^{2}-i \xi x+\eta x+i \xi x^{\prime}+\eta x^{\prime}\right) \psi(t)(x) \overline{\psi(t)\left(x^{\prime}\right)}
$$

and let $I_{\varepsilon}$ be

$$
\int_{\Omega}\left(\int_{\mathbb{R}^{n}}\left(\int_{\mathbb{R}^{n} \times \mathbb{R}^{n}} \Psi_{\varepsilon}\left(x, x^{\prime} ; \xi, \eta\right) \mathrm{d} x \mathrm{~d} x^{\prime}\right) \mathrm{d} \xi\right) \mathrm{d} \eta .
$$

Namely,

$$
I_{\varepsilon}=\int_{\Omega} q(\eta)\left(\int_{\mathbb{R}^{n}} e^{-\varepsilon \xi^{2}}|(\mathfrak{F} \psi(t))(\xi+i \eta)|^{2} \mathrm{~d} \xi\right) \mathrm{d} \eta .
$$

Since

$$
\int_{\mathbb{R}^{n}} e^{-\varepsilon \xi^{2}}|(\mathfrak{F} \psi(t))(\xi+i \eta)|^{2} \mathrm{~d} \xi \nearrow \int_{\mathbb{R}^{n}}|(\mathfrak{F} \psi(t))(\xi+i \eta)|^{2} \mathrm{~d} \xi
$$


as $\varepsilon \searrow 0, \lim _{\varepsilon \downarrow 0} I_{\varepsilon}$ coincides with $I$.

As $\Psi_{\varepsilon} \in L^{1}\left(\mathbb{R}^{4 n}\right)$,

$$
I_{\varepsilon}=\int_{\Omega}\left(\int_{\mathbb{R}^{n}}\left(\int_{\mathbb{R}^{n}}\left(\int_{\mathbb{R}^{n}} \Psi_{\varepsilon}\left(x, x^{\prime} ; \xi, \eta\right) \mathrm{d} \xi\right) \mathrm{d} x^{\prime}\right) \mathrm{d} x\right) \mathrm{d} \eta
$$

by Fubini's theorem. Since

$$
\begin{aligned}
\int_{\mathbb{R}^{n}} \exp \left(-\varepsilon \xi^{2}\right) e^{-i \xi \cdot\left(x-x^{\prime}\right)} \mathrm{d} \xi & =(\pi / \varepsilon)^{n / 2} \exp \left(-\frac{1}{4 \varepsilon}\left(x-x^{\prime}\right)^{2}\right) \\
& =(2 \pi)^{n} \overline{W_{\varepsilon}\left(x-x^{\prime}\right)}
\end{aligned}
$$

we have

$$
\begin{aligned}
\int_{\mathbb{R}^{n}}\left(\int_{\mathbb{R}^{n}} \Psi_{\varepsilon}\left(x, x^{\prime} ; \xi, \eta\right) \mathrm{d} \xi\right) \mathrm{d} x^{\prime} & =\int_{\mathbb{R}^{n}} q(\eta) e^{\eta \cdot\left(x+x^{\prime}\right)} \overline{W_{\varepsilon}\left(x-x^{\prime}\right) \cdot \psi(t)\left(x^{\prime}\right)} \cdot \psi(t)(x) \mathrm{d} x^{\prime} \\
& =q(\eta) \overline{W_{\varepsilon} *(g(\eta, t))(x)}(g(\eta, t))(x),
\end{aligned}
$$

where $(g(\eta, t))(x)=e^{\eta \cdot x} \cdot(\psi(t))(x)$. By Young's inequality the absolute value of

$$
\left\langle g(\eta, t), W_{\varepsilon} * g(\eta, t)\right\rangle_{L^{2}}
$$

is dominated by

$$
\begin{aligned}
\|g(\eta, t)\|_{L^{2}}^{2} & =\int_{\mathbb{R}^{n}}|(g(\eta, t))(x)|^{2} \mathrm{~d} x \\
& =\int_{\mathbb{R}^{n}} e^{2 \eta \cdot x}|\phi(x)|^{2} \mathrm{~d} x .
\end{aligned}
$$

Since $q(\cdot)|g|^{2}(\cdot, t) \in L^{1}\left(\Omega \times \mathbb{R}^{n}\right)$ and

$$
\int_{\mathbb{R}^{n}}\left(\int_{\Omega} q(\eta)|(g(\eta, t))(x)|^{2} \mathrm{~d} \eta\right) \mathrm{d} x=\int_{\mathbb{R}^{n}}\left(\mathfrak{L}_{\Omega} q\right)(x)|\phi(x)|^{2} \mathrm{~d} x,
$$

we have

$$
\begin{aligned}
\int_{\Omega} q(\eta)\|g(\eta, t)\|_{L^{2}}^{2} \mathrm{~d} \eta & =\int_{\mathbb{R}^{n}}\left(\int_{\Omega} q(\eta)|(g(\eta, t))(x)|^{2} \mathrm{~d} \eta\right) \mathrm{d} x \\
& =\int_{\mathbb{R}^{n}}\left(\mathfrak{L}_{\Omega} q\right)(x)|\phi(x)|^{2} \mathrm{~d} x .
\end{aligned}
$$

We also obtain

$$
\begin{aligned}
\int_{\Omega} p(\eta)\|g(\eta, t)\|_{L^{2}}^{2} \mathrm{~d} \eta & =\int_{\mathbb{R}^{n}}\left(\int_{\Omega} p(\eta)|(g(\eta, t))(x)|^{2} \mathrm{~d} \eta\right) \mathrm{d} x \\
& =\int_{\mathbb{R}^{n}}\left(\mathfrak{L}_{\Omega} p\right)(x)|\phi(x)|^{2} \mathrm{~d} x .
\end{aligned}
$$

12 
in the same way. Therefore $\|g(\eta, t)\|_{L^{2}}<\infty$ for almost every $\eta$ where $p(\eta)$ is not zero. Hence

$$
W_{\varepsilon} * g(\eta, t) \longrightarrow g(\eta, t) \quad \text { in } L^{2} \quad \text { as } \varepsilon \rightarrow 0
$$

for almost every $\eta$ with $p(\eta) \neq 0$. This yields

$$
\left\langle g(\eta, t), W_{\varepsilon} * g(\eta, t)\right\rangle_{L^{2}} \longrightarrow\|g(\eta, t)\|_{L^{2}} \quad \text { as } \varepsilon \rightarrow 0
$$

for the same $\eta$ above. Thus the estimate

$$
\left|q(\eta)\left\langle g(\eta, t), W_{\varepsilon} * g(\eta, t)\right\rangle_{L^{2}}\right| \leqq p(\eta)\|g(\eta, t)\|_{L^{2}}^{2}
$$

with Lebesgue's convergence theorem yields

$$
\begin{aligned}
I_{\varepsilon} & =\int_{\Omega} q(\eta)\left\langle g(\eta, t), W_{\varepsilon} * g(\eta, t)\right\rangle_{L^{2}} \mathrm{~d} \eta \\
& \longrightarrow \int_{\Omega} q(\eta)\|g(\eta, t)\|_{L^{2}}^{2} \mathrm{~d} \eta \\
& =\int_{\mathbb{R}^{n}}\left(\mathfrak{L}_{\Omega} q\right)(x)|\phi(x)|^{2} \mathrm{~d} x \quad \text { as } \varepsilon \rightarrow 0 .
\end{aligned}
$$

Therefore

$$
I=\int_{\mathbb{R}^{n}}\left(\mathfrak{L}_{\Omega} q\right)(x)|\phi(x)|^{2} \mathrm{~d} x .
$$

This completes the proof.

Remark 5 By setting $p(x)=q(x)=(2 \pi a)^{-n / 2} \exp \left(-x^{2} /(2 a)\right)$ and $\Omega=\mathbb{R}^{n}$, Theorem 1 reduces to Theorem 2 .

Remark 6 The shape of $\Omega$ in Theorem 2 can be relaxed into a star-shaped domain with respect to the origin. For each $x \in \Omega$ there is a bounded open convex set containing $x$ and the origin. This together with Lemma 4 yields the existence of an analytic continuation $u$ of $U(t) \phi$ defined on $\mathbb{R}^{n}+i t \Omega$ satisfying (2.1) in view of the uniqueness theorem of analytic continuations. By the same argument as above we have the conclusion.

Corollary 1 Let $a>0$ and suppose that

$$
\left(\prod_{j=1}^{n} \frac{\sinh 2 a x_{j}}{2 a x_{j}}\right)^{1 / 2} \phi \in L^{2}
$$

Then, 
(i) $U(t) \phi$ with $t>0$ has an analytic continuation $u(t, \cdot)$ defined on $\mathbb{R}^{n}+i Q_{a t}$, where

$$
Q_{a t}=(-a t, a t)^{n}=\left\{\eta=\left(\eta_{1}, \ldots, \eta_{n}\right) ;-a t<\eta_{j}<a t, j=1, \ldots, n\right\} .
$$

(ii) $u(t, \cdot)$ with $t>0$ satisfies the following identity.

$$
\begin{aligned}
& (2 a t)^{-n} \int_{Q_{a t}}\left(\int_{\mathbb{R}^{n}}\left|\exp \left(-i \frac{(\xi+i \eta)^{2}}{2 t}\right) u(t, \xi+i \eta)\right|^{2} \mathrm{~d} \xi\right) \mathrm{d} \eta \\
= & \sum_{\alpha \geq 0} \frac{(2 a)^{2|\alpha|}}{\prod_{j=1}^{n}\left(2 \alpha_{j}+1\right) !}\left\|J^{\alpha} U(t) \phi\right\|_{L^{2}}^{2} \\
= & \int_{\mathbb{R}^{n}}\left(\prod_{j=1}^{n} \frac{\sinh \left(2 a x_{j}\right)}{2 a x_{j}}\right)|\phi(x)|^{2} \mathrm{~d} x .
\end{aligned}
$$

Proof. Let $p(x)=\left|Q_{a}\right|^{-1}$. Then

$$
\int_{Q_{a}} p(y) e^{2 x \cdot y} \mathrm{~d} y=\prod_{j=1}^{n} \frac{\sinh 2 a x_{j}}{2 a x_{j}}=\sum_{\alpha \geq 0} \frac{(2 a)^{2|\alpha|}}{\prod_{j=1}^{n}\left(2 \alpha_{j}+1\right) !} x^{2 \alpha} .
$$

We have thus shown the corollary.

Remark 7 We have some other examples for $\Omega, p, q, \mathfrak{L}_{\Omega} p$ and $\mathfrak{L}_{\Omega} q$ in Theorem 2 as follows:

(i)

$$
\begin{aligned}
\Omega & =Q_{\pi}=(-\pi, \pi)^{n} \\
p(x) & =\prod_{j=1}^{n}\left|\sin N_{j} x_{j}\right|, \\
q(x) & =\prod_{j=1}^{n} \sin N_{j} x_{j}, \\
\left(\mathfrak{L}_{\Omega} p\right)(x) & =\prod_{j=1}^{n} \frac{\sinh 2 \pi x_{j}}{\tanh \left(\pi x_{j} / N_{j}\right)} \cdot \frac{2 N_{j}}{4 x^{2}+N_{j}^{2}}, \\
\left(\mathfrak{L}_{\Omega} q\right)(x) & =\prod_{j=1}^{n} \frac{2(-1)^{N_{j}+1} N_{j} \sinh 2 \pi x_{j}}{4 x_{j}^{2}+N_{j}^{2}} .
\end{aligned}
$$


(ii)

$$
\begin{aligned}
\Omega & =(-\pi, \pi)^{n} \\
p(x) & =\prod_{j=1}^{n}\left|\cos N_{j} x_{j}\right|, \\
q(x) & =\prod_{j=1}^{n} \cos N_{j} x_{j}, \\
\left(\mathfrak{L}_{\Omega} p\right)(x) & =\prod_{j=1}^{n} \frac{2 \sinh 2 \pi x_{j}}{4 x_{j}^{2}+N_{j}^{2}}\left(2 x_{j}+\frac{N_{j}}{\sinh \pi x_{j} / N_{j}}\right), \\
\left(\mathfrak{L}_{\Omega} q\right)(x) & =\prod_{j=1}^{n} \frac{4(-1)^{N_{j}} x_{j} \sinh 2 \pi x_{j}}{4 x_{j}^{2}+N_{j}^{2}} .
\end{aligned}
$$

Here $N_{j} \in \mathbb{N}$ for $j=1, \ldots, n$. The proof depends on a direct calculation. Details are omitted.

\section{References}

[AHS] Hiroaki Aikawa, Nakao Hayashi and Saburou Saitoh: Isometrical identities for the Bergman and the Szegö spaces on a sector, J. Math. Soc. Japan 43(1991), 195-201.

[C] Thierry Cazenave: Semilinear Schrödinger Equations, Courant lecture notes in mathematics 10, American Mathematical Society, Providence, 2003.

[DS] Nelson Dunford and Jacob T. Schwartz: Linear Operators, Part I, General Theory, Pure and Applied Mathematics 7, Interscience, New York, 1958.

[HK1] Nakao Hayashi and Keiichi Kato: Regularity in time of solutions to nonlinear Schrödinger equations, Journal of Functional Analysis 128(1995), 253-277.

[HK2] Nakao Hayashi and Keiichi Kato: Analyticity in time and smoothing effect of solutions to nonlinear Schrödinger equations, Commun. Math. Phys. 184(1997), 273-300.

[HO] Nakao Hayashi and Tohru Ozawa: On the derivative nonlinear Schrödinger equation, Physica D 55(1992), 14-36.

[HS1] Nakao Hayashi and Saburou Saitoh: Analyticity and smoothing effect for the Schrödinger equation, Ann. Inst. Henri Poincaré, Physique théorique 52(1990), 163-173. 
[HS2] Nakao Hayashi and Saburou Saitoh: Analyticity and global existence of small solutions to some nonlinear Schrödinger equations, Commun. Math. Phys. 129(1990), 27-41.

[KM] Tosio Kato and Kyūya Masuda: Nonlinear evolution equations and analyticity. I., Ann. Inst. Henri Poincaré, Analyse non linéaire 3(1986), 455-467.

[M] Yoshihiro Mizuta: Potential Theory in Euclidean Spaces, Mathematical Sciences and Applications 6, Gakkōtosho, Tokyo, 1996.

[MRZ] Yoshinori Morimoto, Luc Robbiano and Claude Zuily: Remark on the analytic smoothing for the Schrödinger equation, Indiana Univ. Math. J. 49(2000), 1563-1579.

[Nak] Kuniaki Nakamitsu: Analytic finite energy solutions of the nonlinear Schrödinger equation, Commun. Math. Phys. 260(2005), 117-130.

[Nar] Raghavan Narasimhan: Complex Analysis in One Variable, Birkhäuser, Boston, 1985.

[OYY] Tohru Ozawa, Kazuyuki Yamauchi and Yasuko Yamazaki: Analytic smoothing effect for solutions to Schrödinger equations with nonlinearity of integral type, Osaka J. Math. 42 (2005), 737-750.

[RZ1] Luc Robbiano and Claude Zuily: Microlocal analytic smoothing effect for the Schrödinger equation, Duke Math. J. 100(1999), 93-129.

[RZ2] Luc Robbiano and Claude Zuily: Analytic theory for the quadratic scattering wave front set and application to the Schrödinger equation, Astérisque 283, Societe mathematique de France, Paris, 2002.

[S] Saburou Saitoh: Theory of Reproducing Kernels and Its Applications, Pitman Research Notes in Mathematics Series 189, Longman Scientific and Technical, Harlow, 1988.

[SS] Catherine Sulem and Pierre-Louis Sulem: The Nonlinear Schrödinger Equation : Self-Focusing and Wave Collapse, Springer, New York, 1999.

[T1] Hideki Takuwa: Analytic smoothing effects for a class of dispersive equations, Tsukuba J. Math. 28(2004), 1-34.

[T2] Hideki Takuwa: Microlocal analytic smoothing effects for operators of real principal type, Osaka J. Math. 43(2006), 13-62. 